\title{
Increased systemic inflammation in children with Down syndrome
}

\author{
Dean Huggard a,b,c,h,*, Lynne Kelly ${ }^{\mathrm{a}, \mathrm{b}}$, Emer Ryan ${ }^{\mathrm{a}, \mathrm{b}}$, Fiona McGrane ${ }^{\mathrm{a}, \mathrm{c}}$, Niamh Lagan ${ }^{\mathrm{a}, \mathrm{c}}$, \\ Edna Roche ${ }^{\mathrm{a}, \mathrm{c}}$, Joanne Balfe ${ }^{\mathrm{a}, \mathrm{c}}$, T. Ronan Leahy ${ }^{\mathrm{a}, \mathrm{f}}$, Orla Franklin ${ }^{\mathrm{a}, \mathrm{g}}$, Derek G. Doherty ${ }^{\mathrm{a}, \mathrm{b}}$, \\ Eleanor J. Molloy a,b,c,d,e,h
}

${ }^{a}$ Paediatrics, Trinity College, The University of Dublin, Ireland

b Trinity Translational Medicine Institute (TTMI), Trinity College Dublin, Ireland

c Paediatrics, Children's Hospital Ireland (CHI) at Tallaght, Tallaght University Hospital, Ireland

${ }^{\mathrm{d}}$ Coombe Women and Infants University Hospital, Ireland

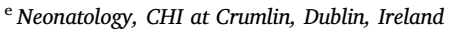

${ }^{\mathrm{f}}$ Immunology, CHI at Crumlin, Dublin, Ireland

${ }^{g}$ Cardiology, CHI at Crumlin, Dublin, Ireland

${ }^{\mathrm{h}}$ National Children's Research Centre, CHI at Crumlin, Dublin, Ireland

\section{A R T I C L E I N F O}

\section{Keywords:}

Down syndrome

Inflammation

Cytokines

Innate immunity

\begin{abstract}
A B S T R A C T
Children with Down syndrome (DS) develop more infections, have an increased mortality from sepsis and an increased incidence of chronic inflammatory conditions. Cytokine dysregulation may underpin these clinical sequelae and raised pro-inflammatory biomarkers are a feature in adults with DS. The importance of the antiinflammatory mediators IL-1ra and IL-10, as well as cytokines Epo and VEGF, which could impact on the pathogenesis and outcomes in congenital heart disease (CHD) which is more prevalent in DS, are less well known. We examined a comprehensive array of pro-(IL-2, IL-6, IL-8, IL-18, IL-1 $\beta$, TNF- $\alpha$, IFN- $\gamma$ ), and anti-inflammatory (IL-10 and IL-1ra) mediators, cytokines involved in inflammation in response to hypoxia (EPO), propagating angiogenesis (VEGF), and myelopoiesis (GM-CSF), by enzyme linked immunosorbent assay (ELISA), as well as discussing the potential impact of significant CHD and Lipopolysaccharide endotoxin on these mediators. 114 children with DS and 60 age and sex matched controls were recruited. Children with Down syndrome exhibit significantly greater levels of pro and anti-inflammatory cytokines; IL-2, IL-6, IL-10, IL-1ra, as well as increased Epo, VEGF and GM-CSF at baseline. CHD does not seem to have an impact on circulating cytokines beyond the acute surgical phase. Both cohorts had similar responses to LPS stimulation. These differences may contribute to varied clinical outcomes, acutely like in sepsis, and over time in autoimmunity.
\end{abstract}

\section{Introduction}

Down syndrome (DS) is the most common chromosomal anomaly and results from extra genetic chromosome 21 material, affecting approximately 1 in 700 births in the USA [1]. DS is associated with several co-morbidities including, congenital heart disease, developmental delay, obstructive sleep apnoea, gastrointestinal atresias, and abnormal myelopoiesis [2]. Dysregulation of the immune system is another common feature of DS, including lower T and B cell numbers [3,4], a smaller thymus gland, reduced regulatory $\mathrm{T}$ lymphocytes and suboptimal antibody responses to immunisation [5-7]. Although altered serum cytokines in DS $[8,9]$ have been described, these mainly evaluate pro-inflammatory mediators in older cohorts.

Children with DS develop more infections, are more likely to be admitted to hospital with respiratory tract infections (RTI) [10], and have a 30\% increased mortality from sepsis [11]. In addition, children with DS have an increased incidence of chronic inflammatory conditions and autoimmunity [12]. A recent meta-analysis evaluating the cytokine profile in DS showed increased pro-inflammatory mediators IL- $1 \beta$, TNF- $\alpha$ and IFN- $\gamma$ [8]. In sepsis there is significant cytokine release which can lead to an acute systemic inflammatory response (SIRS), which may be protective but if prolonged or potentiated may be deleterious. Crucial to a good outcome is a controlled dampening of the immune response. However, a prolonged compensatory anti-

\footnotetext{
Abbreviations: DS, Down syndrome; CHD, congenital heart disease; SIRS, systemic inflammatory response syndrome; CARS, compensatory anti-inflammatory response syndrome; RTI, respiratory tract infection; TAM, transient abnormal myelopoiesis

* Corresponding author at: Trinity Centre for Health Sciences, Tallaght University Hospital, Dublin 24, Ireland.

E-mail address: huggardd@tcd.ie (D. Huggard).
} 
inflammatory response (CARS) may lead to an immunodeficient state and poorer outcomes [13]. Therefore, understanding the inflammatory response to LPS is important in children with DS. IL-10 and IL-1ra are two anti-inflammatory cytokines that are implicated in fine tuning the balance between SIRS and CARS. Children with DS have increase mortality in the context of sepsis, and perhaps it is an imbalance of these signals which leads to poorer clinical outcomes.

Approximately $50 \%$ of all children with DS have cardiovascular disease [14]. Cytokines important in responding to hypoxia or promoting new blood vessel growth like EPO and VEGF could have significant impacts in the pathogenesis of this disease and potentially on outcomes. Indeed, children with DS have better post cardiac surgery outcomes compared with patients without DS [15]. Pulmonary hypertension is also commonly seen in children with DS [16]; and both Epo and VEGF have been associated with this disorder $[17,18]$. GM-CSF is another vascular cytokine which mediates its effects by promoting white blood cell production. It has been linked to the development of transient abnormal myelopoiesis (TAM) in DS, which affects around $10 \%$ of infants in this cohort, and can ultimately lead to myelodysplasia and acute leukaemia [19].

We examined the anti-inflammatory signals (IL-10 and IL-1ra), a comprehensive array of inflammatory cytokines (IL-2, IL-6, IL-8, IL-18, IL-1 $\beta$, TNF- $\alpha$, IFN- $\gamma$ ), cytokines involved in inflammation in response to hypoxia (EPO) propagating angiogenesis (VEGF) and myelopoiesis (GM-CSF), as well as discussing the potential clinical impact of significant congenital heart disease in DS, and LPS endotoxin on these mediators.

\subsection{Methods}

\subsubsection{Study population}

This project was approved by the Ethics Committees of Children's Health Ireland (CHI) at Tallaght (Ref: 2017-05 [1]; approved 18.05.17) and CHI at Crumlin Dublin (Ref: GEN/565/17; approved 05.05.17), Ireland. All families and participants received verbal and documented information on the study and written consent was obtained prior to recruitment. The following children were enrolled: (a) Children with Down syndrome $<16$ years old attending the multidisciplinary Down syndrome clinic in CHI at Tallaght or at Crumlin and (b) Age-matched Paediatric Controls: children attending for phlebotomy or day case procedures and whose results were subsequently normal. Children in both groups were excluded if they had recent fever or evidence of infection.

\subsubsection{Sample preparation}

Blood samples (1-3 mL) for in vitro experiments were collected in a sodium citrate anti-coagulated blood tube and analysed within two hours of phlebotomy. Whole blood was incubated at $37{ }^{\circ} \mathrm{C}$ for $1 \mathrm{~h}$ untreated (vehicle) or with Lipopolysaccharide (LPS; E. coli 0111:B4: SIGMA Life Science, Wicklow, Ireland) $10 \mathrm{ng} / \mathrm{Ml}$. After incubation the samples were centrifuged at $10,000 \mathrm{rpm}$ for $10 \mathrm{~min}$ at room temperature. The plasma supernatant was stored at $-80{ }^{\circ} \mathrm{C}$ for subsequent analysis.

\subsubsection{Multiplex ELISA}

The following cytokines were evaluated using the MSD ${ }^{\circledR}$ MULTISPOT assay system from Mesoscale (MSD Diagnostics, USA): tumour necrosis factor alpha (TNF- $\alpha$ ), interleukin-1 $\beta$ (IL-1 $\beta$ ), interleukin-2 (IL2 ), interleukin-6 (IL-6), interleukin-8 (IL-8), interferon- $\gamma$ (IFN- $\gamma$ ), interleukin-18 (IL-18), vascular endothelial growth factor (VEGF), erythropoietin (Epo), interleukin-1 receptor antagonist (IL-1ra), and interleukin-10 (IL-10). Extracted peripheral blood plasma, as described above, was transferred to a 96 well MSD plate and these cytokines were assessed as previously outlined [20] and per manufacturer's instructions. Assays were readily transferred to the U-PLEX platform with calibration curves showing expected signals, sensitivity precision, and accuracy. Sensitivities were $<1 \mathrm{pg} / \mathrm{ml}$ for many assays. All assays used the same diluents. Non-specific binding between assays was typically $<0.1 \%$. U-plex sample recovery is within the acceptable range (70-130\%) with samples diluting linearly from 2 to 16 -fold. MesoScale Discovery, Rockville, MD, USA (www.meso-scale.com).

\subsubsection{Statistics}

Statistical analysis was performed using un-paired $t$ tests to compare mean results between two independent cohorts. The KolmogorovSmirnov test was used to check normality. Significance was defined as $\mathrm{p}=0.05$. Results shown are expressed as mean \pm standard error of the mean (SEM) unless otherwise stated. Data was analysed with FloJo software (Oregon, USA) and GraphPad Prism.

\subsection{Results}

\subsubsection{Patient characteristics}

There were 114 children with Down syndrome (DS) enrolled with an age (mean \pm SD) of $5.7 \pm 4.7$ years $(y)$ of which 55 were female $(48.2 \%)$, and the controls $(\mathrm{n}=60)$ with a mean age of $6.9 \pm 4.8 \mathrm{y}$, of which 28 were female $(46.7 \%)$. In the DS cohort, $74 \%(n=84)$ had a diagnosis of congenital heart disease with $39 \%(n=33)$ of these requiring surgery in infancy. The following was the distribution of cardiac lesions in this group ( $\mathrm{n}=33$ ): Atrioventricular septal defect (AVSD) $(n=17)$; Ventricular septal defect (VSD) repair $(n=4)$; Tetralogy of Fallot $(n=4)$; Atrial septal defect (ASD) and VSD repair $(n=3)$; ASD repair $(n=2)$; Patent ductus arteriosus (PDA) coil $(n=1)$. The time from surgery to sampling was $51.1 \pm 22.5$ months. For comparison in the cohort of children with DS we compared those with congenital heart disease (CHD) requiring surgery $(n=33)$ versus those with CHD not requiring surgery $(n=51)$ and those without CHD $(n=30)$.

\subsubsection{Cytokines at baseline in children with DS and controls}

There were significant increases in children with DS versus controls at baseline in the following: Epo, VEGF, GM-CSF, IL-10, IL-1ra, IL-6, IL2 ( $\mathrm{p} \leq 0.05$ ) (Table 1 and Figs. 1-3(a-c), Fig. 4(a-b)). There were no significant differences seen in the levels of IL-1 $\beta$, TNF- $\alpha$, INF- $\gamma$, IL-8 or IL-18 (p > 0.05; Table 1).

Table 1: Interleukin 2 (IL-2); Interleukin 6 (IL-6); Interleukin 8 (IL8); Interleukin 10 (IL-10); Interleukin 18 (IL-18); Interleukin $1 \beta$ (IL-1ß); Tumour necrosis factor $\alpha$ (TNF- $\alpha$ ); Interferon $\gamma$ (IFN- $\gamma$ ); Interleukin 1 receptor antagonist (IL-1ra); Granulocyte macrophage - colony stimulating factor (GM-CSF); Erythropoietin (Epo); Vascular endothelial growth factor (VEGF). Cytokine values in bold denotes $\mathrm{p} \leq 0.05$.

\subsubsection{Effects of LPS on cytokine responses}

Following LPS stimulation there were significant elevation in VEGF, IL-10, IL-1ra, IL-6, IL-2, TNF- $\alpha$, IFN- $\gamma$, IL-8 in both cohorts ( $\mathrm{p} \leq 0.05$;

Table 1

The mean, standard error of the mean (SEM) and p-values of cytokine levels ( $\mathrm{pg} / \mathrm{ml}$ ) in plasma of children with Down syndrome and the control group.

\begin{tabular}{llll}
\hline Cytokine & Controls & DS & p-Value \\
\hline IL-2 & $0.36 \pm 0.07$ & $0.68 \pm 0.11$ & $\mathbf{0 . 0 5}$ \\
IL-6 & $0.67 \pm 0.06$ & $1.88 \pm 0.28$ & $\mathbf{0 . 0 2}$ \\
IL-8 & $5.34 \pm 0.50$ & $8.16 \pm 1.40$ & 0.12 \\
IL-10 & $0.41 \pm 0.06$ & $0.70 \pm 0.57$ & $\mathbf{0 . 0 0 2}$ \\
IL-18 & $529.6 \pm 37.58$ & $508.8 \pm 17.87$ & 0.57 \\
IL-1 $\beta$ & $0.35 \pm 0.06$ & $0.57 \pm 0.08$ & 0.07 \\
TNF- $\alpha$ & $4.72 \pm 0.71$ & $8.97 \pm 3.12$ & 0.33 \\
IFN- $\gamma$ & $24.21 \pm 5.06$ & $22.95 \pm 2.85$ & 0.81 \\
IL-1ra & $331.2 \pm 38.48$ & $497.1 \pm 44.25$ & $\mathbf{0 . 0 1}$ \\
GM-CSF & $0.14 \pm 0.04$ & $0.27 \pm 0.03$ & $\mathbf{0 . 0 2}$ \\
Epo & $67.0 \pm 4.87$ & $116.2 \pm 6.72$ & $<\mathbf{0 . 0 0 0 1}$ \\
VEGF & $88.88 \pm 5.13$ & $181.0 \pm 14.48$ & $<\mathbf{0 . 0 0 0 1}$ \\
\hline
\end{tabular}

The bold denotes $\mathrm{p} \leq 0.05$ or significance. 


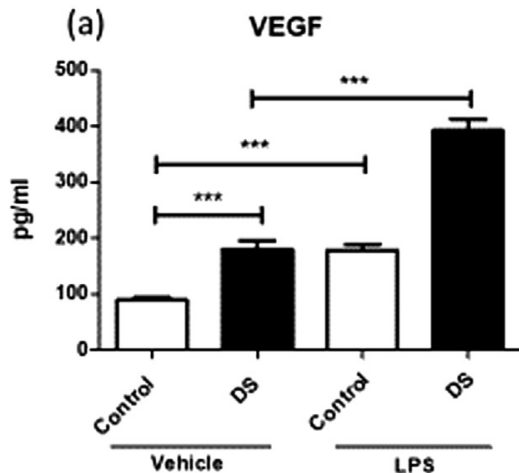

(d)

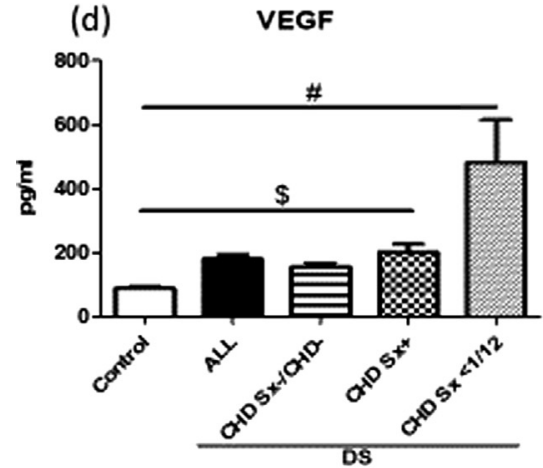

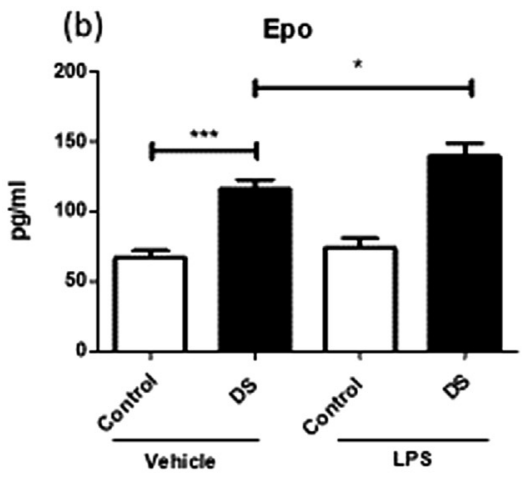

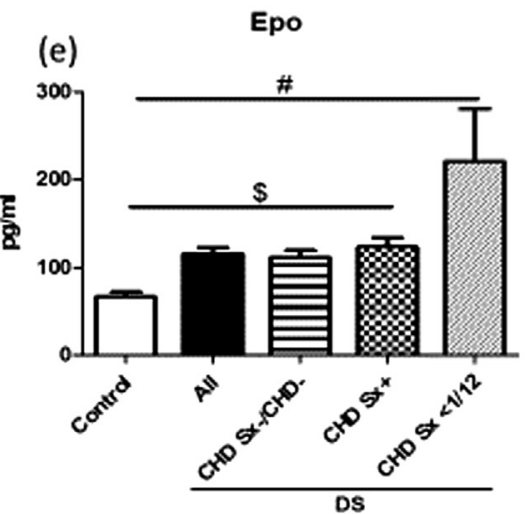

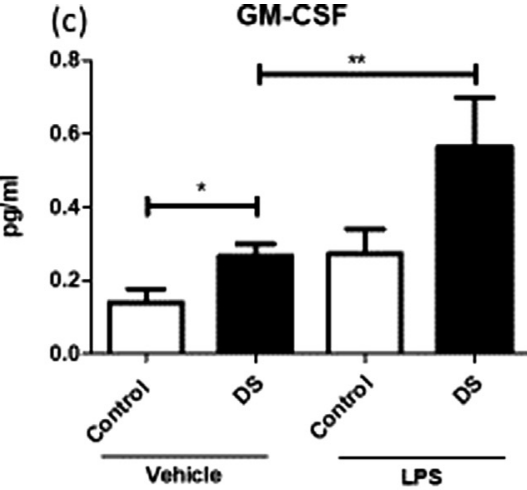

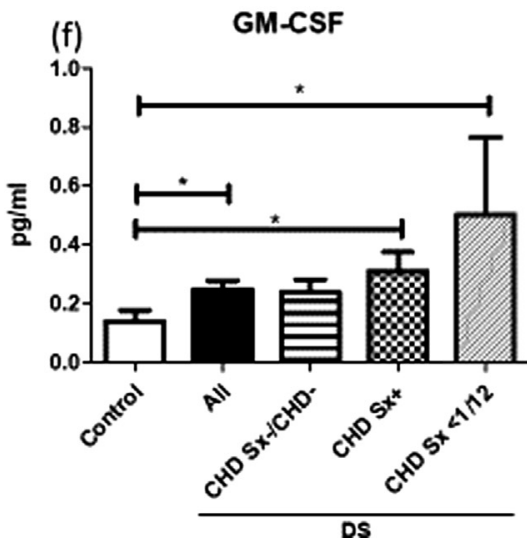

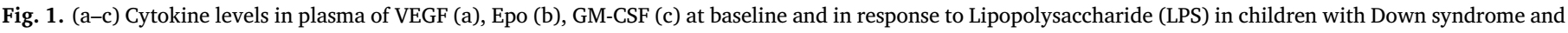

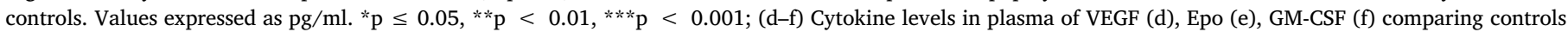

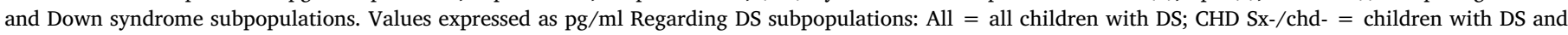

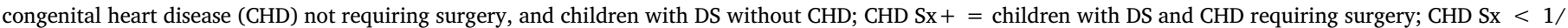

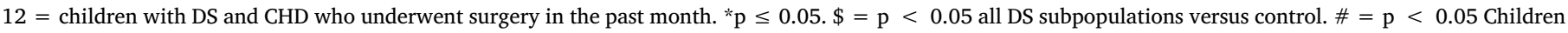
with CHD who underwent surgery in the past month versus the other DS subpopulations and controls.

Figs. 1-3(d-f), Fig. 4(c-d)). In children with DS, EPO and GM-CSF were significantly increased after incubation with LPS, which was not observed for controls (Fig. 1(b) and (c)). There was no response in IL-18 following LPS in either cohort. (Fig. 2(b)).

\subsubsection{CHD and cytokine responses}

To further examine the differences in the cytokine profile in children with DS and CHD the cohort was divided as follows: CHD requiring surgical intervention and CHD not requiring surgery and children without CHD. There were no significant differences in cytokine levels between children with no CHD and those with CHD not requiring surgery.

Comparing the subpopulations with DS and the control group showed that for Epo, VEGF, IL-6, IL-10, IL-1ra, both DS cohorts had significantly higher levels than controls [Figs. 1, 2(d-f) and 4(c-d)]. Children with DS and CHD had significantly higher IL-8 compared with controls, even though the total levels in children with DS were not significantly different for this cytokine [Fig. 3(d)]. GM-CSF was significantly raised in children requiring surgery compared to children who did not require intervention or had no underlying CHD [Fig. 1(f)].

Further analysis demonstrated that for every cytokine the children with DS and CHD had the highest mean value. For children with DS and CHD requiring surgery; Epo, VEGF, IL-6, IL-1ra, IL-1 $\beta$, and IL-8 were significantly greater than for children with DS and CHD not requiring surgery and children without CHD. IL-18, TNF- $\alpha$, and IFN- $\gamma$ were not different in the DS subpopulations irrespective of CHD.

There were no differences in cytokine levels between children with DS with CHD not requiring surgery or no CHD and those with DS and CHD who had surgery more than 1 month ago (Figs. 1-3(d-f) and 4(c-d)) once these infants with recent surgery ( $<1$ month; $n=7$ ) were excluded.

\section{Discussion}

We have demonstrated that children with DS have increased pro and anti-inflammatory cytokine levels compared with age-matched controls; IL-2, IL-6, IL-10, IL-1ra, Epo, VEGF and GM-CSF. Although there is definite immune dysregulation in children with DS $[4,21]$ they had robust LPS responses similar to controls. CHD requiring surgery did not appear to have a significant influence on cytokines in DS except in the acute post-operative period. However, IL- 8 was the exception and was significantly raised in children with DS who required surgical intervention versus controls. Children without significant CHD also still exhibit a significantly elevated cytokine profile compared to controls.

A recent meta-analysis examining circulating serum cytokine levels in patients with DS found significant differences compared to controls. The analysis reviewed 19 studies involving 957 patients with DS and 541 controls, and concluded that significantly higher levels of Il-1 $\beta$, TNF- $\alpha$, IFN- $\gamma$, and neopterin were present in the population with DS [8]. The authors found that circulating levels of IL-4, IL-6, IL- 8 and IL10 were not statistically different between the DS and control groups. However, it was suggested that one study [9] significantly altered the result for IL-6, and when this outlier was omitted from analysis, IL-6 was significantly raised compared with controls. It is worth noting that although results of some cytokines differ from our findings, for example, IL-1 $\beta$, a key pro-inflammatory cytokine implicated in chronic disease [22] almost reached significance in our study ( $\mathrm{p}$ value $=0.07$ ). The population with DS in the meta-analysis was predominantly adults 
(a)

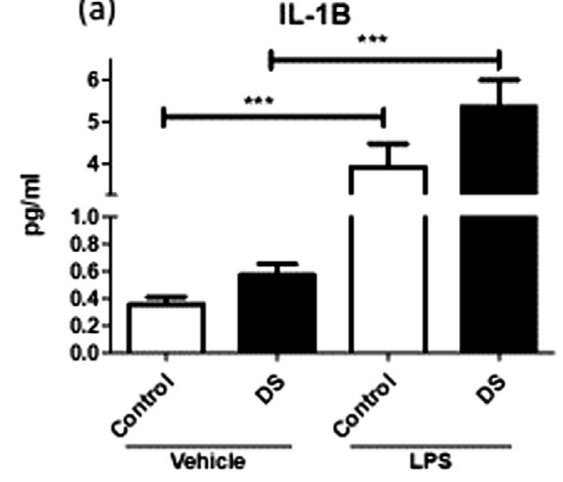

(d)

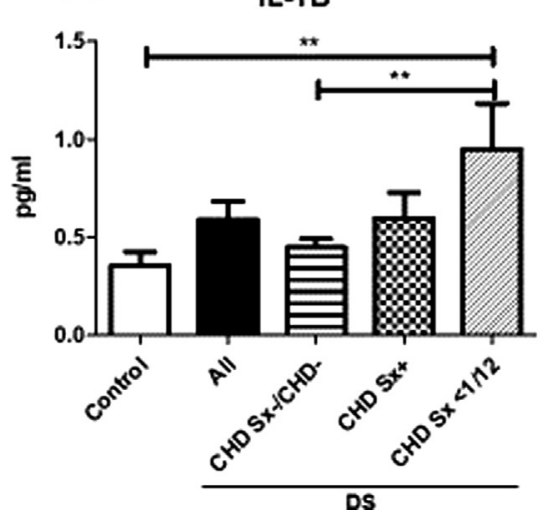

(b)

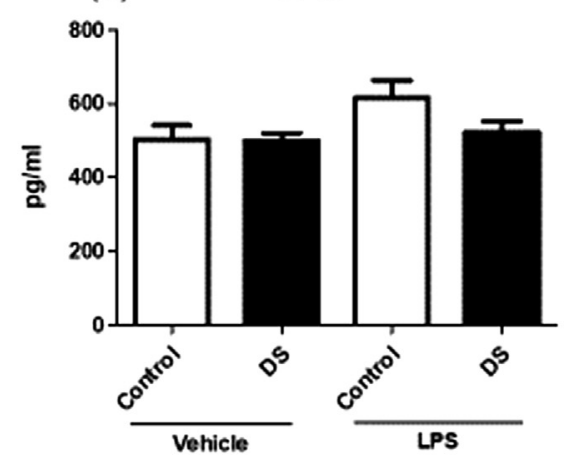

(e)

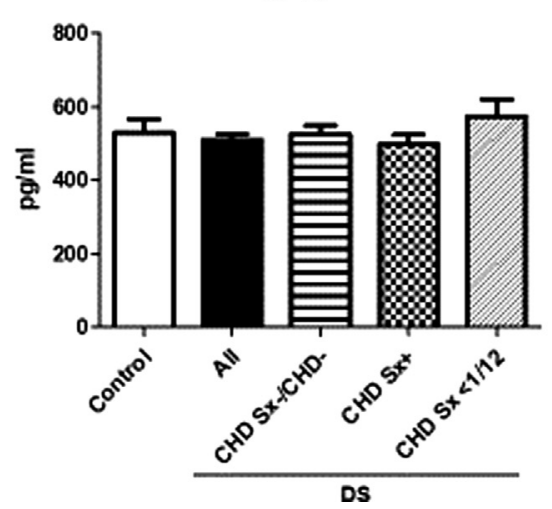

(c)

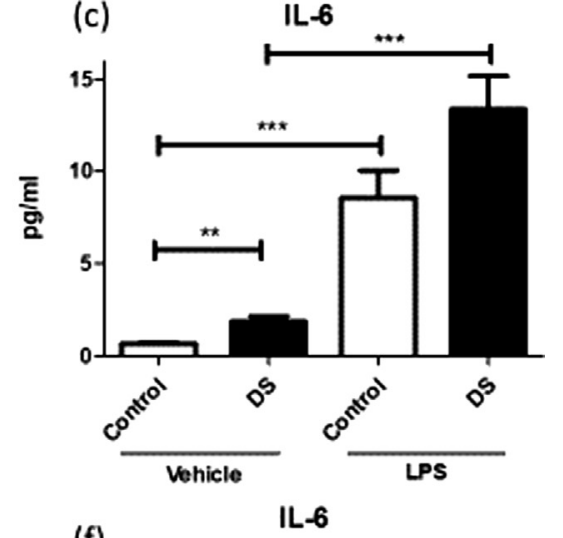

(f)

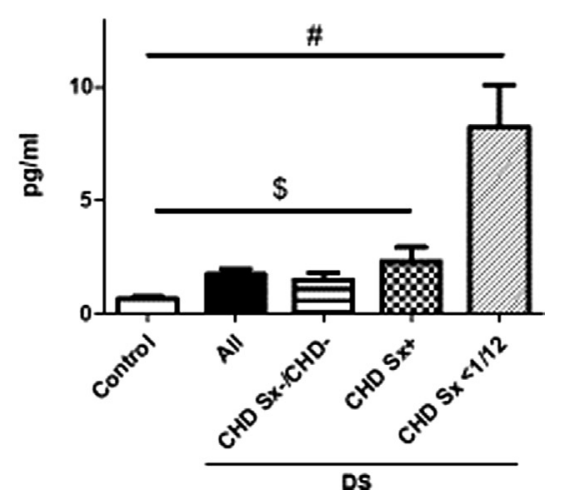

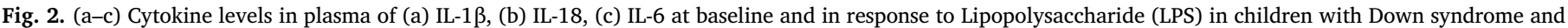

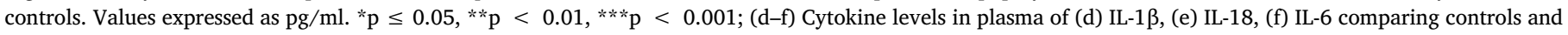

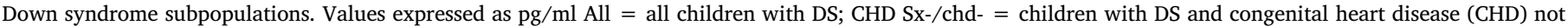

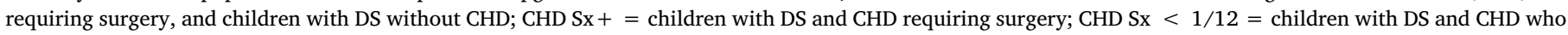

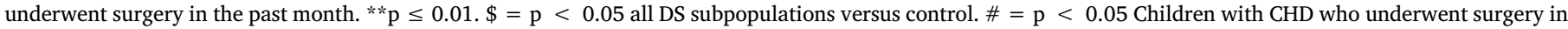
the past month versus the other DS subpopulations and controls.

as 6 studies were included involving adults ( $>16$ mean age), and of the paediatric studies only one study [9] had a mean age lower than in our study. Furthermore, we had the largest cohort described and although there have been other studies evaluating cytokines in DS [8,9,23-25] this paper examines a comprehensive array of cytokines including antiinflammatory and cardiovascular mediators.

Increased levels of VEGF and Epo were significantly greater in children with DS than controls DS, irrespective of CHD. Erythropoietin (EPO) has a crucial role in red blood cell production, however, it is now being recognised as having a myriad of effects such as altering the response to inflammation or injury, inhibiting apoptosis in the setting of hypoxia and thus improving tissue survival [26]. In newborn infants with DS, excess Epo levels in plasma and consequent elevated circulating haemoglobin are reported [27]. Infants $(n=11)$ with persistent pulmonary hypertension of the newborn (PPHN) or cyanotic congenial heart disease (CCHD) had increased hypoxia-inducible factor (HIF)-1 $\alpha$, VEGF and Epo mRNA expression compared to healthy newborns [28]. PPHN and CCHD are seen with increased frequency in DS [29].

We describe increased VEGF levels in our DS cohort. VEGF is an important in regulating angiogenesis, lymphangenesis and is associated with chronic inflammation and tumour growth [30,31]. VEGF-A is a significant mitogen involved in the development of atrioventricular valvular cushions and abnormal expression is associated with CHD [32]. Sanchez et al. [33] found that DS results in abnormal angiogenesis in utero, increased expression of VEGF-A, which is also linked to congenital heart disease lesions. Increased expression of HIF-2 $\alpha$ has been associated with excess VEGF production [34], and HO-1 has been shown to be key in cardiac embryonic development [35]. VEGF is also associated with pulmonary hypertension and this complication as mentioned is seen more frequently in DS [18].

We report significantly elevated GM-CSF levels in children with DS. GM-CSF is implicated in the pathogenesis of transient abnormal myelopoiesis (TAM) in DS. This is a form of leukaemia occurring in infancy, can resolve spontaneously, and may arise from the foetal liver. GM-CSF from stromal tissue of the foetal liver promotes foetal blast proliferation by creating a suitable haematopoietic microenvironment [19]. None of the patients in this study had TAM but may have not been detected as no universal neonatal screening. However, their matched full blood counts and differentials did not have any evidence of TAM at the time of cytokine analysis. There has been associations made with raised GMCSF and poorer neurological outcomes in neonatal encephalopathy [20] and this may implicate this cytokine in adverse neuroinflammation, although the results of a 5 year follow up of ex preterm small for gestational age (SGA) $(n=216)$ children who were randomised to either receive GM-CSF or not, found no neurodevelopmental or adverse outcome differences between groups [36]. GM-CSF is also important to facilitating cross-talk between myeloid cells and $\mathrm{T}$ lymphocytes which if dysregulated can lead to chronic inflammation [37].

Although a pro-inflammatory phenotype in children with DS has been described [38], there is a less information on anti-inflammatory cytokines such as IL-10 and IL-1ra. We demonstrated that both IL-10 and IL-1ra are significantly raised in children with DS. IL- 10 is an antiinflammatory cytokine which reduces inflammation by inhibiting the propagation of pro-inflammatory cytokines such as TNF- $\alpha$ and IL-6 [9]. There are conflicting results regarding IL-10 in cohorts with DS as Nateghi et al. found a lower serum level of IL-10 when compared with controls (DS $n=24$, Controls $n=24$ ), and a reciprocal increased level of the pro-inflammatory cytokine TNF- $\alpha$ and IFN- $\gamma$ [25]. However, 

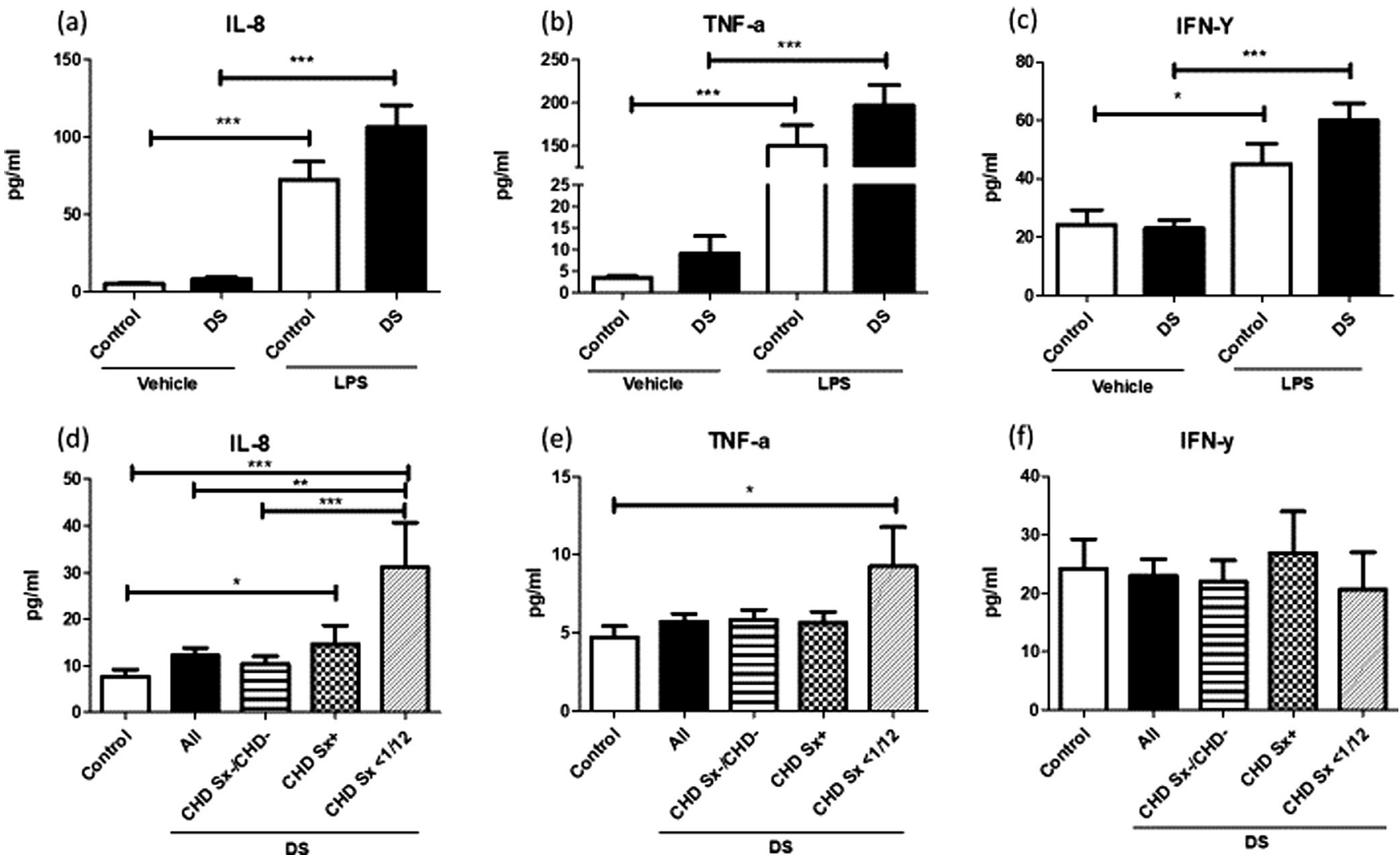

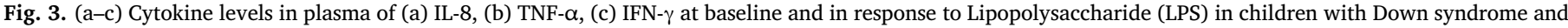

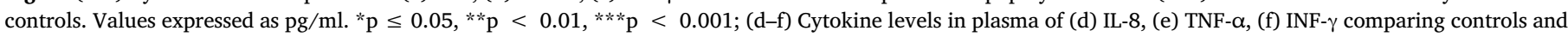

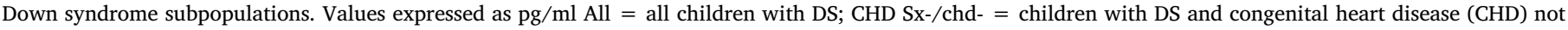

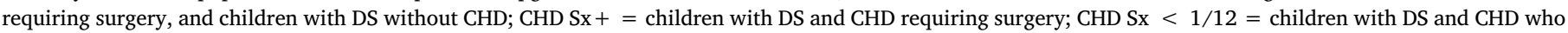

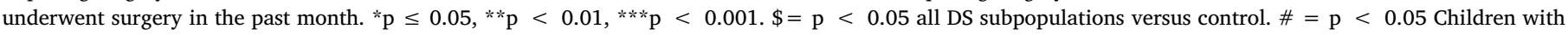
CHD who underwent surgery in the past month versus the other DS subpopulations and controls.

Cetiner et al, reported an increased level of IL-10, and IL-4 in serum and a reduction of TNF- $\alpha$ and IL-6 [9], hypothesising that this anti-inflammatory picture may contribute to the increased level of RTIs seen in childhood. Indeed, Broers et al. [23] described a significant increase in IL-10 after stimulation of whole blood with Streptococcus pneumoniae in children with DS versus their healthy siblings, suggesting a heightened anti-inflammatory response which may be contributory to more severe pneumococcal pneumonia in this cohort. Further research by Guazzarotti et al. (DS $n=24$, Control $n=42$ ), and Iulita et al. (DS $\mathrm{n}=31$, Controls $\mathrm{n}=31$ ) has reported an increase in serum IL-10 in DS versus controls $[39,40]$.

Interleukin 1 receptor antagonist (IL-1ra) is an anti-inflammatory cytokine that abrogates IL-1 signalling, has TNF- $\alpha$ inhibitory effects, and suppresses $\mathrm{T}$ cell responses [41]. Our findings suggest that although pro-inflammatory signals are elevated, so too are the counter-regulatory cytokines IL-10 and IL-1ra. It is a key regulator in maintaining a balance between pro and anti-inflammatory stimuli. Excess IL-1ra has been associated with worse outcomes in the setting of gram-positive arthritis and sepsis. Ali et al. [42] reported more aggressive disease and increased mortality in a murine model of Staphylococcus aureus arthropathy and sepsis. There is a higher incidence of inflammatory arthritis in children with DS [43], and elevated levels of IL-1ra have been shown in adults with rheumatoid arthritis [44].

Interleukin 6 (IL-6) is a key pro-inflammatory mediator released by leucocytes, fibroblasts and endothelial cells. It stimulates the production of IL-1, TNF- $\alpha$ and other pro-inflammatory cytokines to potentiate an inflammatory response [45]. We found greater IL-6 levels in DS, this provides evidence of a hyper-inflammatory response. Clinically, IL- 6 is crucial in mounting an appropriate defence to pathogenic organisms, while if aberrantly produced can lead to chronic inflammation and autoimmunity [46]. This cytokine plays a role in Alzheimer's disease, early onset being ubiquitous in DS, and involves degenerative processes in the central nervous system [47]. Liscastro et al. found elevated IL-6, soluble IL-6 receptors and soluble intracellular adhesion molecule-1 (sICAM-3), soluble vascular adhesion molecule-1 (sVCAM-1) and CRP were produced in children with DS compared to controls [48]. The increase of IL- 6 and CRP from DS children was similar to that found in elderly patients with clinical AD. IL-6 has also been described as significantly raised in response to influenza A virus. An ex vivo cytokine response following stimulation with live Influenza A virus reported the following to be increased in children with DS compared with controls; IL-6, IL-1 $\beta$, IL-8, and TNF- $\alpha$ [24].

We have demonstrated increased IL-2 in children with DS, although studies to date involving IL-2 and DS show no significant difference in this cytokine versus controls $[9,49]$. Interleukin 2 (IL-2) is causes production of $\mathrm{T}$ lymphocytes, memory and effector cells, and has an essential role in the propagation and functionality of regulatory $\mathrm{T}$ cells (Treg cells). The latter important in controlling immunity, inflammation and promoting tolerance. Therefore IL-2 is key in promoting other $\mathrm{T}$ cells to provide an immune response, whilst also enabling Tregs to limit the degree of inflammation [50].

A possible explanation for the elevated cytokine profile demonstrated in children with DS, regardless of CHD, is a dysregulation of Toll like receptor (TLR) signalling. We have recently reported increased TLR2 expression on neutrophils and monocytes and altered gene expression of key regulatory proteins involved in signal propagation [51]. Excess cytokine levels in DS could refelct anomlaous signalling of TLR pathways as the end point of these pathways results in the release of 

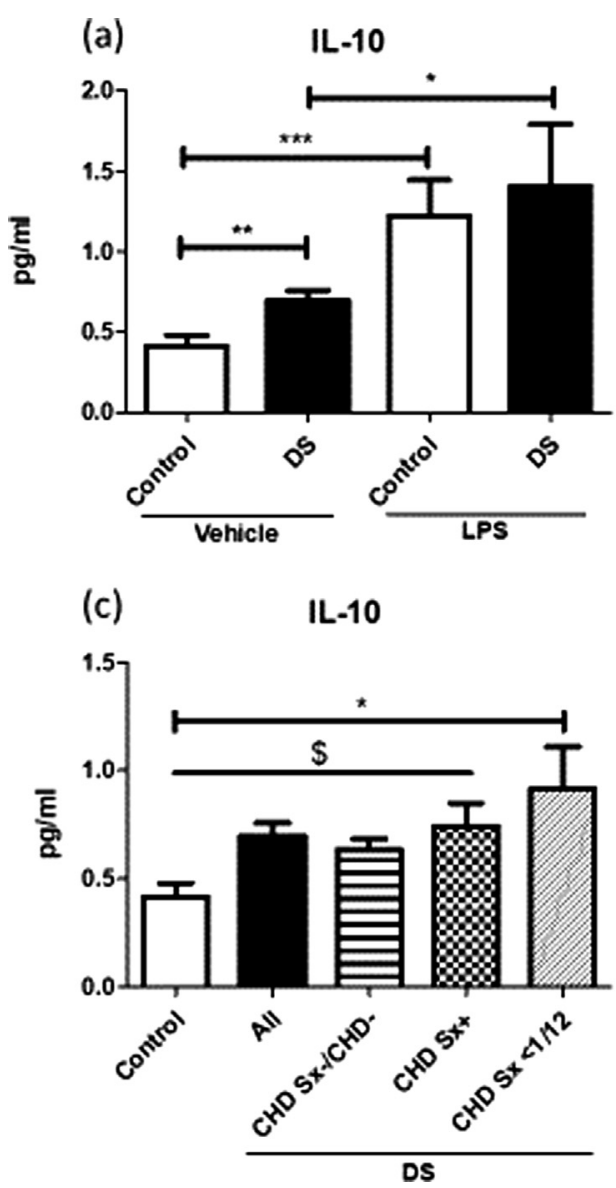

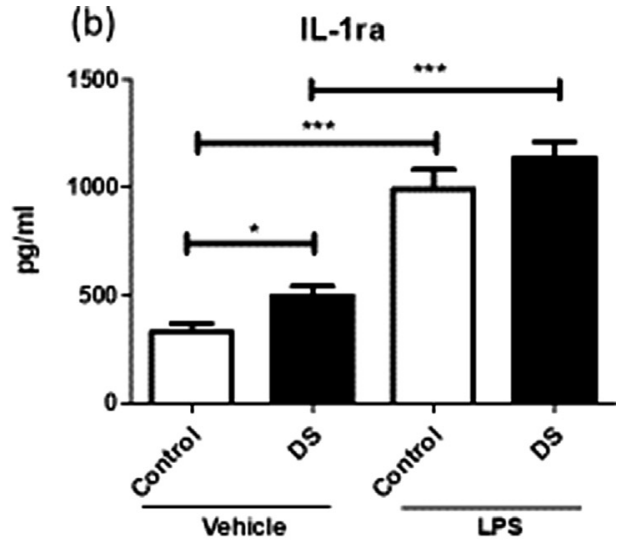

(d)

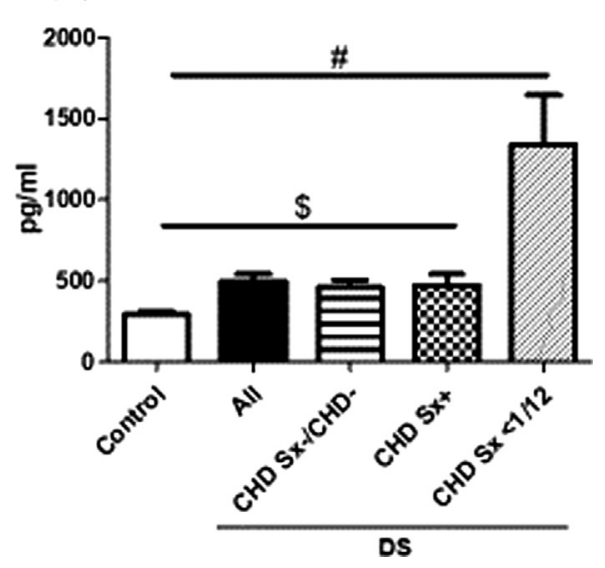

Fig. 4. (a-b) Cytokine levels in plasma of (a) IL-10 and (b) IL-1ra at baseline and in response to Lipopolysaccharide (LPS) in children with Down syndrome and controls. Values expressed as $\mathrm{pg} / \mathrm{ml}$. * $\mathrm{p} \leq 0.05$, $* * \mathrm{p}<0.01, * * * \mathrm{p}<0.0001 ;$ (d-e) Cytokine levels in plasma of (d) IL-10 and (e) IL-1ra comparing controls and Down syndrome subpopulations. Values expressed as $\mathrm{pg} / \mathrm{ml}$. All = all children with DS; CHD Sx-/chd- = children with DS and congenital heart disease (CHD) not requiring surgery, and children with DS without CHD; CHD Sx $+=$ children with DS and CHD requiring surgery; CHD Sx $<1 / 12=$ children with DS and CHD who underwent surgery in the past month. ${ }^{*} \mathrm{p} \leq 0.05 . \$=\mathrm{p}<0.05$ all DS subpopulations versus control. \# $=\mathrm{p}<0.05$ Children with CHD who underwent surgery in the past month versus the other DS subpopulations and controls. inflammatory mediators. Furthermore, children with DS may display a hyperresponsive immune reaction as we have previously reported increased neutrophil activation post LPS, and greater TLR4 on non-classical monocytes [52]. Indeed, a pro-inflammatory phenotype may predominate in this population leading to excess circualting cytokines.

Although this study represents the largest most comprehensive apprasial of cytokines in a paediatric population, there were several limitations. The exact cellular source for the differing cytokine levels between children with DS and controls was not established as whole blood was used. Serial cytokine incubation times with endotoxin would be useful to understan the evolution of the inflammatory response in children with DS especially following cardiac surgery.

\section{Conclusion}

Overall this study demonstrates that children with Down syndrome exhibit significantly greater levels of pro and anti-inflammatory cytokines as well as increased Epo, VEGF and GM-CSF at baseline. From a clinical perspective CHD does not seem to have an impact on circulating cytokines beyond the acute surgical phase. The response to LPS stimulation is largely similar between cases and controls. The differences in circulating cytokines may contribute to varied clinical outcomes, acutely like in sepsis, as the imbalance between SIRS and CARS becomes detrimental, and over time in chronic inflammation. Epo \& VEGF have been associated with vascular remodelling and may be associated with pulmonary hypertension. Given the high incidence of this disorder in our cohort and the excess Epo and VEGF demonstrated, further research is needed to evaluate the role of these mediators in the development of pulmonary hypertension.
Data availability statement

The experimental data used to support the findings of this study are available from the corresponding author upon request.

\section{Authorship statement}

The manuscript is being submitted on behalf of all the authors and is the original work of all authors. DH was responsible for recruitment, sample acquistion, lab experiments, analysis and was responsible for writing the main draft of the manuscript. LK and ER added expert laboratory assisstance. FM, NL, ER, JB, OF were responsible for patient recruitment, sample acquisition, reviewing and editing the manuscript. TR L, DD and EM were key in study design, supervising the research and its outcomes and providing crucial editorial assistance. All authors had editorial license to review and re-draft the manuscript, and all contributors had to approve the final edit. All authors are accountable for the accuracy and scientific integrity of this work.

\section{Funding}

This study was funded by the National Children's Research Centre (NCRC) [14187], Crumlin, Dublin 12 and the National Children's Hospital Fund [206965], Tallaght, Dublin, Ireland

\section{Declaration of Competing Interest}

The authors declare that they have no known competing financial interests or personal relationships that could have appeared to influence the work reported in this paper. 


\section{Appendix A. Supplementary material}

Supplementary data to this article can be found online at https:// doi.org/10.1016/j.cyto.2019.154938.

\section{References}

[1] S.E. Parker, C.T. Mai, M.A. Canfield, R. Rickard, Y. Wang, R.E. Meyer, et al., Updated National Birth Prevalence estimates for selected birth defects in the United States, 2004-2006, Birth Defects Res. A 88 (12) (2010) 1008-1016.

[2] Down's Syndrome Medical Interest Group (DSMIG) (UK \& Ireland), Department of Paediatrics University of Dublin TCTNCsH, Tallaght Hospital. Medical Management of Children \& Adolescents with Down Syndrome in Ireland, 2015.

[3] E. Martinez, D. Castaneda, S. Jaramillo, A. Iregui, T. Quinonez, J.A. Rodriguez, et al., Altered immune parameters correlate with infection-related hospitalizations in children with Down syndrome, Hum. Immunol. 77 (7) (2016) 594-599.

[4] G. Ram, J. Chinen, Infections and immunodeficiency in Down syndrome, Clin. Exp. Immunol. 164 (1) (2011) 9-16.

[5] M.A. Kusters, N.C. Manders, B.A. de Jong, R.W. van Hout, G.T. Rijkers, E. de Vries, Functionality of the pneumococcal antibody response in Down syndrome subjects, Vaccine 31 (52) (2013) 6261-6265.

[6] M.A. Kusters, Jol-Van Der, E.C. Zijde, R.H. Gijsbers, E. de Vries, Decreased response after conjugated meningococcal serogroup $\mathrm{C}$ vaccination in children with Down syndrome, Pediatr. Infect. Dis. J. 30 (9) (2011) 818-819.

[7] M.A. Kusters, C.M. Jol-van der Zijde, M.J. van Tol, W.E. Bolz, L.A. Bok, M. Visser, et al., Impaired avidity maturation after tetanus toxoid booster in children with Down syndrome, Pediatr. Infect. Dis. J. 30 (4) (2011) 357-359.

[8] Y. Zhang, M. Che, J. Yuan, Y. Yu, C. Cao, X.Y. Qin, et al., Aberrations in circulating inflammatory cytokine levels in patients with Down syndrome: a meta-analysis, Oncotarget 8 (48) (2017) 84489-84496.

[9] S. Cetiner, O. Demirhan, T.C. Inal, D. Tastemir, Y. Sertdemir, Analysis of peripheral blood T-cell subsets, natural killer cells and serum levels of cytokines in children with Down syndrome, Int. J. Immunogenet. 37 (4) (2010) 233-237.

[10] J.M. Hilton, D.A. Fitzgerald, D.M. Cooper, Respiratory morbidity of hospitalized children with Trisomy 21, J. Paediatr. Child Health 35 (4) (1999) 383-386.

[11] M.M. Garrison, H. Jeffries, D.A. Christakis, Risk of death for children with down syndrome and sepsis, J. Pediatr. 147 (6) (2005) 748-752.

[12] F.P. Pellegrini, M. Marinoni, V. Frangione, A. Tedeschi, V. Gandini, F. Ciglia, et al., Down syndrome, autoimmunity and T regulatory cells, Clin. Exp. Immunol. 169 (3) (2012) 238-243.

[13] B. Mathias, B.E. Szpila, F.A. Moore, P.A. Efron, L.L. Moldawer, A review of GM-CSF therapy in sepsis, Medicine 94 (50) (2015) e2044.

[14] P.F. Faria, J.A. Nicolau, M.Z. Melek, Nde S. de Oliveira, B.E. Bermudez, R.M. Nisihara, Association between congenital heart defects and severe infections in children with Down syndrome, Rev. Port Cardiol. 33 (1) (2014) 15-18.

[15] J.M. Evans, M. Dharmar, E. Meierhenry, J.P. Marcin, G.W. Raff, Association between Down syndrome and in-hospital death among children undergoing surgery for congenital heart disease: a US population-based study, Circulat. Cardiovasc. Qual. Outcomes 7 (3) (2014) 445-452.

[16] T. Martin, A. Smith, C.R. Breatnach, E. Kent, I. Shanahan, M. Boyle, et al., Infants born with Down syndrome: burden of disease in the early neonatal period, J. Pediatr. 193 (2018) 21-26.

[17] V.A. Karamanian, M. Harhay, G.R. Grant, H.I. Palevsky, W.E. Grizzle, R.T. Zamanian, et al., Erythropoietin upregulation in pulmonary arterial hypertension, Pulmonary Circulat. 4 (2) (2014) 269-279.

[18] J. Pako, A. Bikov, K. Karlocai, G. Csosza, L. Kunos, G. Losonczy, et al., Plasma VEGF levels and their relation to right ventricular function in pulmonary hypertension, Clin. Exp. Hypertens. (New York, NY: 1993) 37 (4) (2015) 340-344.

[19] J. Miyauchi, H. Kawaguchi, Fetal liver stromal cells support blast growth in transient abnormal myelopoiesis in Down syndrome through GM-CSF, J. Cell. Biochem. 115 (6) (2014) 1176-1186.

[20] F.M. O'Hare, R.W. Watson, A. O'Neill, R. Segurado, D. Sweetman, P. Downey, et al., Serial cytokine alterations and abnormal neuroimaging in newborn infants with encephalopathy, Acta Paediatr. 106 (4) (2017) 561-567.

[21] M.A. Kusters, R.H. Verstegen, E.F. Gemen, E. de Vries, Intrinsic defect of the immune system in children with Down syndrome: a review, Clin. Exp. Immunol. 156 (2) (2009) 189-193.

[22] J. Tozser, S. Benko, Natural compounds as regulators of NLRP3 inflammasomemediated IL-1beta production, Mediators Inflamm. 2016 (2016) 5460302.

[23] C.J. Broers, R.J. Gemke, S.A. Morre, M.E. Weijerman, A.M. van Furth, Increased production of interleukin-10 in children with Down syndrome upon ex vivo stimulation with Streptococcus pneumoniae, Pediatr. Res. 75 (1-1) (2014) 109-113.

[24] C.J. Broers, R.J. Gemke, M.E. Weijerman, K.F. van der Sluijs, A.M. van Furth, Increased pro-inflammatory cytokine production in Down Syndrome children upon stimulation with live influenza A virus, J. Clin. Immunol. 32 (2) (2012) 323-329.

[25] M. Nateghi Rostami, M. Douraghi, A. Miramin Mohammadi, B. Nikmanesh, Altered serum pro-inflammatory cytokines in children with Down's syndrome, Eur. Cytokine Netw. 23 (2) (2012) 64-67.

[26] C. French, Erythropoietin in critical illness and trauma, Crit. Care Clin. 35 (2) (2019) 277-287.
[27] J.A. Widness, S.M. Pueschel, J.C. Pezzullo, G.K. Clemons, Elevated erythropoietin levels in cord blood of newborns with Down's syndrome, Biol. Neonate 66 (1) (1994) 50-55.

[28] M.L. Lemus-Varela, M.E. Flores-Soto, R. Cervantes-Munguia, B.M. Torres-Mendoza, G. Gudino-Cabrera, V. Chaparro-Huerta, et al., Expression of HIF-1 alpha, VEGF and EPO in peripheral blood from patients with two cardiac abnormalities associated with hypoxia, Clin. Biochem. 43 (3) (2010) 234-239.

[29] P.S. Shah, J. Hellmann, I. Adatia, Clinical characteristics and follow up of Down syndrome infants without congenital heart disease who presented with persistent pulmonary hypertension of newborn, J. Perinat. Med. 32 (2) (2004) 168-170.

[30] M. Zbucka-Kretowska, K. Charkiewicz, J. Goscik, S. Wolczynski, P. Laudanski, Maternal plasma angiogenic and inflammatory factor profiling in foetal Down syndrome, PLoS ONE 12 (12) (2017) e0189762.

[31] B.A. Hesser, X.H. Liang, G. Camenisch, S. Yang, D.A. Lewin, R. Scheller, et al., Down syndrome critical region protein 1 (DSCR1), a novel VEGF target gene that regulates expression of inflammatory markers on activated endothelial cells, Blood 104 (1) (2004) 149-158.

[32] C. Ackerman, A.E. Locke, E. Feingold, B. Reshey, K. Espana, J. Thusberg, et al., An excess of deleterious variants in VEGF-A pathway genes in Down-syndrome-associated atrioventricular septal defects, Am. J. Hum. Genet. 91 (4) (2012) 646-659.

[33] O. Sánchez, C. Domínguez, A. Ruiz, I. Ribera, J. Alijotas, L. Cabero, et al., Angiogenic gene expression in Down syndrome fetal hearts, Fetal Diagn. Ther. 40 (1) (2016) 21-27.

[34] J.D. Gordan, J.A. Bertout, C.J. Hu, J.A. Diehl, M.C. Simon, HIF-2alpha promotes hypoxic cell proliferation by enhancing c-myc transcriptional activity, Cancer Cell 11 (4) (2007) 335-347.

[35] S. Druyan, A. Cahaner, C.M. Ashwell, The expression patterns of hypoxia-inducing factor subunit alpha-1, heme oxygenase, hypoxia upregulated protein 1 , and cardiac troponin T during development of the chicken heart, Poult. Sci. 86 (11) (2007) 2384-2389.

[36] N. Marlow, T. Morris, P. Brocklehurst, R. Carr, F. Cowan, N. Patel, et al., A randomised trial of granulocyte-macrophage colony-stimulating factor for neonatal sepsis: childhood outcomes at 5 years, Arch. Dis. Child. Fetal Neonatal Ed. 100 (4) (2015) F320-F326.

[37] B. Becher, S. Tugues, M. Greter, GM-CSF: from growth factor to central mediator of tissue inflammation, Immunity 45 (5) (2016) 963-973.

[38] J. Zhao, H. Shang, X. Cao, Y. Huang, X. Fang, S. Zhang, et al., Association of polymorphisms in TLR2 and TLR4 with asthma risk: an update meta-analysis, Medicine 96 (35) (2017) e7909.

[39] L. Guazzarotti, D. Trabattoni, E. Castelletti, B. Boldrighini, L. Piacentini, P. Duca, et al., T lymphocyte maturation is impaired in healthy young individuals carrying trisomy 21 (Down syndrome), Am. J. Intellect. Dev. Disabilit. 114 (2) (2009) $100-109$.

[40] M.F. Iulita, A. Ower, C. Barone, R. Pentz, P. Gubert, C. Romano, et al., An inflammatory and trophic disconnect biomarker profile revealed in Down syndrome plasma: relation to cognitive decline and longitudinal evaluation, Alzheimer's Dementia: J. Alzheimer's Assoc. 12 (11) (2016) 1132-1148.

[41] C. Thompson, R. Davies, E. Choy, Anti cytokine therapy in chronic inflammatory arthritis, Cytokine 86 (2016) 92-99.

[42] A. Ali, M. Na, M.N. Svensson, M. Magnusson, A. Welin, J.C. Schwarze, et al., IL-1 receptor antagonist treatment aggravates staphylococcal septic arthritis and sepsis in mice, PLoS ONE 10 (7) (2015) e0131645.

[43] C. Foley, O.G. Killeen, Musculoskeletal anomalies in children with Down syndrome: an observational study, Arch. Dis. Child. (2018).

[44] S. Ramirez-Perez, U. De la Cruz-Mosso, J. Hernandez-Bello, G.E. Martinez-Bonilla, M.G. Ramirez-Duenas, A.L. Pereira-Suarez, et al., High expression of interleukine-1 receptor antagonist in rheumatoid arthritis: association with IL1RN*2/2 genotype, Autoimmunity 50 (8) (2017) 468-475.

[45] S. Rose-John, K. Winthrop, L. Calabrese, The role of IL-6 in host defence against infections: immunobiology and clinical implications, Nat. Rev. Rheumatol. 13 (7) (2017) 399-409.

[46] T. Tanaka, T. Kishimoto, Targeting interleukin-6: all the way to treat autoimmune and inflammatory diseases, Int. J. Biol. Sci. 8 (9) (2012) 1227-1236.

[47] M.G. Carta, P. Serra, A. Ghiani, E. Manca, M.C. Hardoy, G.S. Del Giacco, et al., Chemokines and pro-inflammatory cytokines in Down's syndrome: an early marker for Alzheimer-type dementia? Psychother. Psychosom. 71 (4) (2002) 233-236.

[48] F. Licastro, M. Chiappelli, M. Ruscica, V. Carnelli, M.M. Corsi, Altered cytokine and acute phase response protein levels in the blood of children with Downs syndrome: relationship with dementia of Alzheimer's type, Int.. J. Immunopathol. Pharmacol. 18 (1) (2005) 165-172.

[49] M.B. Trotta, J.B. Serro Azul, M. Wajngarten, S.G. Fonseca, A.C. Goldberg, J.E. Kalil, Inflammatory and Immunological parameters in adults with Down syndrome, Immun. Age.: I \& A 8 (1) (2011) 4.

[50] A.K. Abbas, E. Trotta, D.R. Simeonov, A. Marson, J.A. Bluestone, Revisiting IL-2: biology and therapeutic prospects, Sci. Immunol. 3 (25) (2018).

[51] D. Huggard, W.J. Koay, L. Kelly, F. McGrane, E. Ryan, N. Lagan, et al., Altered tolllike receptor signalling in children with Down syndrome, Mediat. Inflamm. 2019 (2019) 4068734.

[52] D. Huggard, F. McGrane, N. Lagan, E. Roche, J. Balfe, T.R. Leahy, et al., Altered endotoxin responsiveness in healthy children with Down syndrome, BMC Immunol. 19 (1) (2018) 31. 